\title{
Nitrogen-doped magnetic carbon nanoparticles as catalyst supports for efficient recovery and recycling $\dagger$
}

\author{
Hyeonseok Yoon, Sungrok Ko and Jyongsik Jang* \\ Received (in Cambridge, UK) 15th November 2006, Accepted 15th January 2007 \\ First published as an Advance Article on the web 5th March 2007 \\ DOI: 10.1039/b616660a
}

Palladium nanoparticles were deposited with high dispersion and stability on nitrogen-doped magnetic carbon nanoparticles by a simple impregnation method, and their catalytic performance was investigated for Heck, Suzuki, and Sonogashira coupling reactions.

Although homogeneous catalysts have many advantages over their heterogeneous counterparts, the majority of industrial catalysts consist of metal or metal compound particles dispersed on an appropriate support. ${ }^{1}$ The use of catalyst supports provides effective opportunities for separation of the reaction product and the catalyst. ${ }^{2}$ Of a wide range of catalyst supports, in particular, carbon materials have been extensively employed in heterogeneous catalysis due to their features such as chemical inertness, good mechanical stability, and electrical conductivity. ${ }^{3}$ Since the supports can strongly affect catalyst activity, numerous studies have been performed to modify the carbon supports in an effort to improve catalyst performance. First, advances in the synthesis of materials have made it possible to prepare carbon nanostructures with well-defined morphologies (e.g., surface area and porosity). Typically, carbon nanotubes and mesoporous carbon materials have been highlighted as promising catalyst supports. ${ }^{4}$ Secondly, chemical modification of the carbon surface has been explored to stabilize catalyst-support interactions. Functional groups including hydroxyls, carboxyls and amines were mostly introduced via ozonolysis, plasma activation, acid or base treatment, and so forth. ${ }^{5}$ However, these approaches may deteriorate the mechanical/electronic properties of carbon materials, and the functional groups generated are often unstable under certain catalysis conditions.

Recently, doping of heteroatoms into carbon materials has offered new opportunities for tailoring their chemical/physical properties. The most notable example is nitrogen-doped carbon materials. Rao and co-workers achieved good yields of nitrogendoped carbon nanotubes by pyrolysis of pyridine and nickel phthalocyanine-thiophene (nitrogen content of 3-10 wt \%). ${ }^{6}$ In addition, Mokaya and co-workers fabricated nitrogen-doped carbons in the form of nanotubes and mesoporous structures by using acetonitrile as a carbon precursor (nitrogen content of 6-9 $\left.\mathrm{wt}^{\mathrm{O}} \%\right)^{7}$ Importantly, the nitrogenated sites in the carbon

Hyperstructured Organic Materials Research Center and School of Chemical and Biological Engineering, Seoul National University,

Shinlimdong 56-1, Seoul, 151-742, Korea.

E-mail: jsjang@plaza.snu.ac.kr; Fax: 822888 1604; Tel: 8228807069

$\dagger$ Electronic supplementary information (ESI) available: 1. Electron diffraction pattern of $\mathrm{Pd} / \mathrm{N}-\mathrm{MCNPs}$; 2. Control experiments (TEM images, XRD patterns, and textural parameters of Pd-decorated carbon blacks). See DOI: 10.1039/b616660a matrix would provide strong catalyst-support interactions in anchoring catalyst particles onto the carbon support without the aid of any functionalization processes. ${ }^{8}$

We have previously reported on the fabrication of several types of carbon nanomaterials using nanosized polymer precursors. ${ }^{9}$ Notably, magnetically functionalized carbon nanotubes and nanoparticles could be readily obtained via the carbonization of iron-impregnated polymer nanomaterials. ${ }^{10,11}$ Herein, we investigate the formation of palladium nanoparticles onto nitrogendoped magnetic carbon nanoparticles (N-MCNPs), and their catalytic activity was also examined for several coupling reactions.

First, magnetic carbon nanoparticles were prepared using irondoped polypyrrole nanoparticles as the carbon precursor (carbonization temperature, $800{ }^{\circ} \mathrm{C}$ ). ${ }^{11}$ The resulting carbon nanoparticles include nitrogen atoms in the carbon matrix because the pyrrole repeating unit consists of one nitrogen, four carbons, and five hydrogens: the CHNS elemental analysis indicated that the as-prepared carbon nanoparticles had a nitrogen content of $c a$. $4-5 \mathrm{wt} \%$. It is also important to note that the N-MCNPs are characterized as a microporous nanoarchitecture. To deposit palladium onto the N-MCNPs, $0.1 \mathrm{~g}$ of N-MNCPs was dispersed in $0.8 \mathrm{~mL}$ of water, and subsequently $0.76 \mathrm{~mL}$ of $2.5 \mathrm{wt} \%$ aqueous $\mathrm{Pd}\left(\mathrm{NO}_{3}\right)_{2}$ solution was added in the solution at $25{ }^{\circ} \mathrm{C}$. After drying, the samples were calcined at $300{ }^{\circ} \mathrm{C}$ in air, and then reduced at $300{ }^{\circ} \mathrm{C}$ in a hydrogen atmosphere.

The resulting palladium-loaded N-MNCPs (Pd/N-MCNPs) were observed by transmission electron microscope (TEM). As shown in Fig. 1(a), the diameter of N-MCNPs used as the catalyst

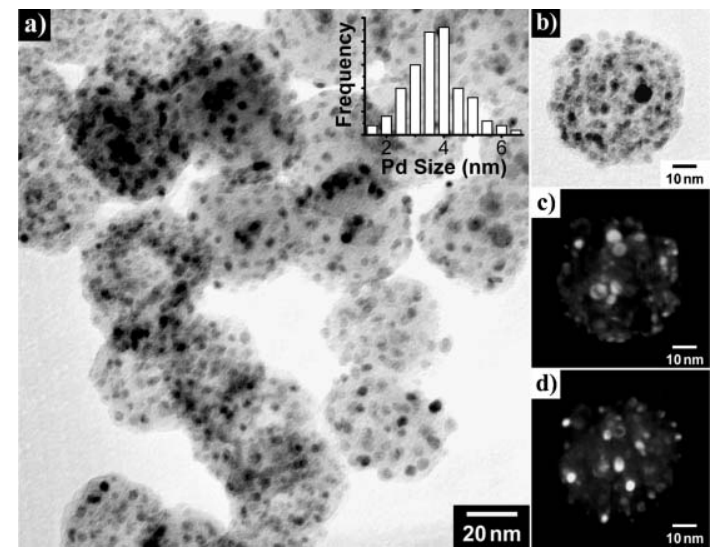

Fig. 1 TEM images of Pd/N-MCNPs: (a) low-magnification bright-field image (inset: size distribution histogram of the Pd nanoparticles formed), (b) high-magnification bright-field image for a single nanoparticle, and the corresponding dark-field images obtained from (c) $\mathrm{Pd}(111)$ and (d) $\operatorname{Pd}(200)$ reflections (for electron diffraction image see ESI, Fig. S1†). 
support was approximately $50 \mathrm{~nm}$. The palladium nanoparticles were well-dispersed onto the N-MCNPs and were not agglomerated. The size distribution of palladium nanoparticles was reasonably narrow, and the average diameter was determined as $3.8 \mathrm{~nm}$ by counting 100 nanoparticles in the TEM image (Fig. 1(a) inset). The dark-field TEM images revealed the (111) and (200) crystallographic planes of the deposited palladium nanoparticles (Fig. 1(c), (d)). The powder X-ray diffraction (XRD) pattern of the Pd/N-MCNPs displayed the characteristic (111), (200), (220) and (311) peaks of the fcc palladium crystal structure (Fig. 2). The average size of the palladium crystallites calculated from the line broadening of the $\operatorname{Pd}(111)$ peak using the Scherrer formula was $4.6 \mathrm{~nm}$, which was commensurable to the result obtained from the TEM observation. Thermogravimetric analysis showed that the $\mathrm{Pd} / \mathrm{N}-\mathrm{MCNPs}$ contained an extremely high palladium content (approximately $40 \mathrm{wt} \%$ ). In general, employing a substantial amount of metal precursor for such a high metal loading commonly gives rise to agglomeration of metal nanoclusters during the reduction process. However, in our case, the excellent dispersion of palladium nanoparticles with narrow size distribution could be achieved over the sample through a simple impregnation method without using any specific ligands or stabilizers. Moreover, the palladium nanoparticles deposited were retained without deformation even after sonication for 5-10 min, which indicated strong binding of the palladium nanoclusters to the carbon support. These results can be explained in the following three ways: (i) the well-defined spherical nanostructures may allow a rapid diffusion rate and wetting of the metal precursor on their surface compared with irregularly shaped spheres, mesoporous channel structures, and tubular nanostructures. (ii) The porous surface of the carbon support can offer steric restriction to confine the metal precursor and to prevent growth of metal clusters due to the surface tension of the metal precursor solution. (iii) The nitrogenated sites are capable of providing a strong metal-support interaction to anchor efficiently metal precursor on the carbon support and to stabilize the final metal nanoclusters.

To confirm these explanations, two different types of carbon blacks containing no nitrogen were employed for control experiments. One was a Vulcan XC72R (a commercially available carbon black, Cabot Corporation), and the other was a carbon black prepared by thermal decomposition of methane using dc thermal plasma processing (Plasnix Co.). These materials consist of

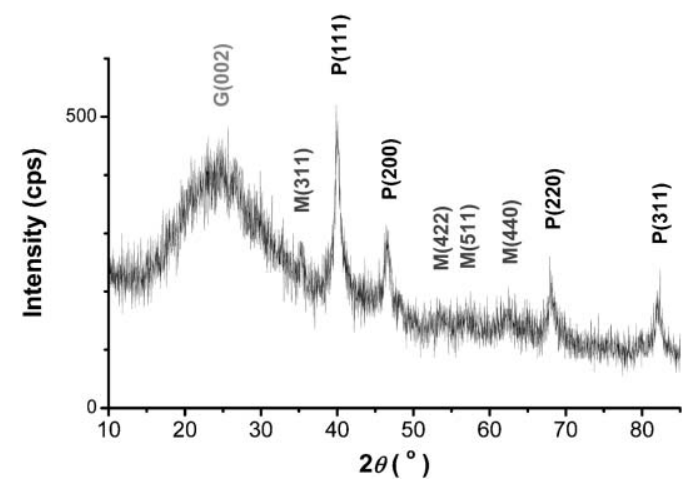

Fig. 2 Powder XRD pattern of Pd/N-MCNPs (G: graphite, $\mathbf{M}: \gamma-\mathrm{Fe}_{2} \mathrm{O}_{3}$, P: palladium). The XRD peaks were collected in the range $2 \theta=10-85^{\circ}$ with a scan speed of $3^{\circ} \mathrm{min}^{-1}(\mathrm{Cu}-\mathrm{K} \alpha$ radiation source: $\lambda=1.5406 \AA)$. aggregates of spherical particles with the diameter of individual particles ranging from 10 to $200 \mathrm{~nm}$. Under the same deposition condition, these carbon blacks could be only decorated with larger agglomerates of palladium nanoparticles that were poorly dispersed on the surface: the palladium size distributions were 2-28 and 2-20 nm, respectively (Fig. S2 and S3, ESI $\dagger$ ). This experimental result may be a good evidence that the well-defined spherical porous nanostructures and the nitrogen doping contribute to the uniform anchoring of palladium nanoparticles with similar sizes.

The magnetic properties of $\mathrm{Pd} / \mathrm{N}-\mathrm{MCNPs}$ were investigated using a superconducting quantum interference device magnetometer (Fig. 3(a)). The saturation of the magnetization could be observed with applied magnetic fields above $15 \mathrm{kOe}(1 \mathrm{kOe}=$ $0.1 \mathrm{~T}$ ), and the saturation magnetization from the hysteresis loop was found to be $6.9 \mathrm{emu} \mathrm{g}^{-1}$. The magnetic property of the carbon support is useful for efficient recovery and recycling of nanocatalysts in liquid-phase reactions. ${ }^{12}$ As an example, when the $\mathrm{Pd} / \mathrm{N}$ MCNPs were dispersed in water giving a black suspension (Fig. 3(b)), upon applying an external magnetic field, the black powder was readily harvested and the solution became transparent (Fig. 3(c)).

To investigate the catalytic activity of the Pd/N-MCNPs, three different types of coupling reactions were performed, as summarized in Table 1. As a typical example for Heck reaction, iodobenzene and styrene were selected as a non-activated aryl halide and an aromatic olefin, respectively (entry 1). In general, non-activated aryl halides have relatively low conversion yields compared with activated aryl halides (e.g., 4-iodoanisole and 2-iodotoluene). Nevertheless, the Heck olefination of iodobenzene with styrene showed high yields of more than $97 \%$ when employing the Pd/N-MCNPs. The yield of the reaction with a commercial palladium/carbon catalyst was only 53\% after $24 \mathrm{~h}$ under the same experimental conditions. ${ }^{13}$ The Suzuki coupling of phenylboronic acid and iodothiophene was also catalyzed using
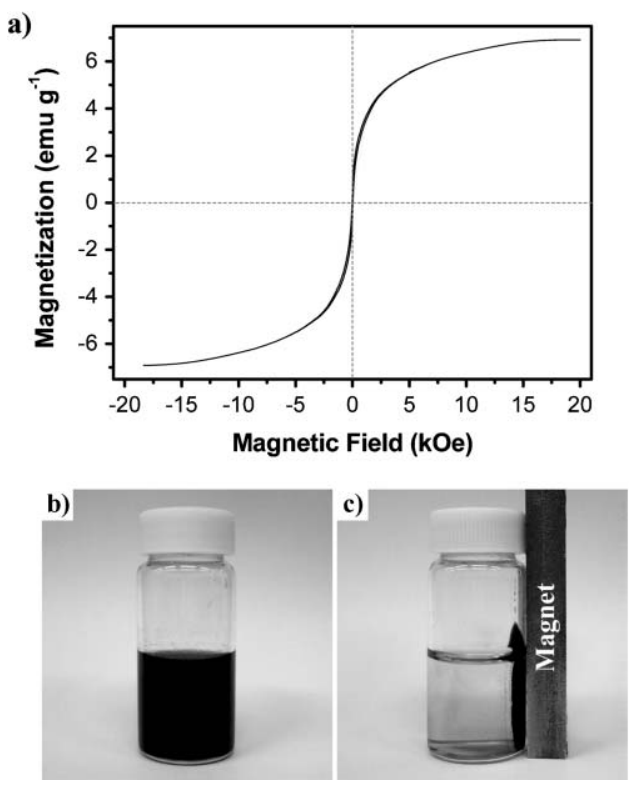

Fig. 3 Magnetic property of Pd/N-MCNPs: (a) hysteresis loop measured at $300 \mathrm{~K}$, and photographs of an aqueous solution containing $0.01 \mathrm{wt} \%$ $\mathrm{Pd} / \mathrm{N}-\mathrm{MCNPs}$ (b) before and (c) after magnetic separation. 
Table 1 Catalytic activities of Pd/N-MCNPs for Heck, Suzuki and Sonogashira coupling reactions

\begin{tabular}{llllll} 
Entry & Reaction & Reagent 1 & Yield $^{e}(\%)$ & 1st cycle 2nd cycle 3rd cycle \\
\hline $3^{b}$ & Seck & Reagent 2 &
\end{tabular}

${ }^{a}$ All reactions were catalyzed using $0.01 \mathrm{~g}$ of Pd/N-MNCPs. ${ }^{b}$ Reaction carried out in $20 \mathrm{~mL}$ of dimethylacrylonitrile with triethylamine (4.46 mmol), iodobenzene $(1.95 \mathrm{mmol})$, and styrene $(2.11 \mathrm{mmol}) .{ }^{c}$ Reaction performed in $50 \mathrm{~mL}$ of ethanol with 4-bromoacetophenone $(3.00 \mathrm{mmol})$, phenylboronic acid $(6.00 \mathrm{~mol})$, 2-iodothiophene $(3.00 \mathrm{mmol})$, and $\mathrm{K}_{3} \mathrm{PO}_{4}(12.00 \mathrm{mmol})$. ${ }^{d}$ Reaction carried out in $20 \mathrm{~mL}$ of dimethyl sulfoxide with $\mathrm{Na}_{2} \mathrm{CO}_{3}(14.47 \mathrm{mmol})$, 4-bromoacetophenone $(10.00 \mathrm{mmol})$, phenylacetylene $(13.33 \mathrm{mmol})$, and $\mathrm{CuI}(0.15 \times$ $\left.10^{-1} \mathrm{mmol}\right) .{ }^{e}$ The yield was calculated by gas chromatography analysis.

the $\mathrm{Pd} / \mathrm{N}-\mathrm{MCNPs}$ (entry 2). A previous report showed that $0.3 \mathrm{~mol} \%$ palladium nanoparticles ( $3.6 \mathrm{~nm}$ in diameter) stabilized by poly( $N$-vinyl-2-pyrrolidone) gave a yield of $78 \%$ after $12 \mathrm{~h}$ for the same reaction. ${ }^{14}$ Although only $1.2 \mathrm{~mol} \%$ palladium content was used under our experimental conditions, high yields of approximately $94 \%$ could be achieved after only $3 \mathrm{~h}$. Finally, the $\mathrm{Pd} / \mathrm{N}-\mathrm{MCNPs}$ were employed to promote a Sonogashira reaction of 4-bromoacetophenone and phenylacetylene as aryl halide and alkyne, respectively (entry 3 ). The desired products were obtained in high yields of more than $91 \%$ with $0.4 \mathrm{~mol} \%$ palladium content for $3 \mathrm{~h}$ at $100{ }^{\circ} \mathrm{C}$. This is comparable to the result ( $92 \%$ yield) obtained using $2 \mathrm{~mol} \%$ palladium/nickel nanoparticles $(3.9 \mathrm{~nm}$ in diameter) for $2 \mathrm{~h}$ at $80{ }^{\circ} \mathrm{C} .{ }^{15}$ Thus, the $\mathrm{Pd} / \mathrm{N}-\mathrm{MCNPs}$ gave high conversion yields for all the three different reactions. Monodisperse spherical carbon nanoparticles allow the easy diffusion of reactants and highly dispersed palladium nanoparticles offer more catalytically active sites for reactants, which may be responsible for the remarkable catalytic activities. It was also noteworthy that the $\mathrm{Pd} / \mathrm{N}-\mathrm{MNCP}$ could be readily reused several times without losing their catalytic activity. If the bonds between metal and support were weak, the metal nanoparticles would be detached from the support. Consequently, it is believed that the strong interaction between palladium clusters and N-MNCPs plays a significant role in preventing metal leaching during the reactions.

In conclusion, we demonstrated the use of N-MCNPs as a magnetically recoverable catalyst support. Well-dispersed palladium nanoparticles with narrow size distribution could be deposited on the N-MCNPs by a simple impregnation method. The Pd/N-MCNPs displayed high catalytic activities for three types of coupling reactions, and the magnetic property of the support gave an opportunity for easy recovery and recycling of the catalyst via magnetic separation. Importantly, versatile metal nanoparticles may be decorated on the N-MCNPs, which might be applied to catalysts, sensors, bactericides, and so forth.

This work was supported by the Brain Korea 21 program of the Korean Ministry of Education, and the Hyperstructured Organic
Materials Research Center is supported by the Korea Science and Engineering Foundation.

\section{Notes and references}

1 J. Grunes, J. Zhu and G. A. Somorjai, Chem. Commun., 2003, 2257; D. J. Cole-Hamilton, Science, 2003, 299, 1702.

2 D. Astruc, F. Lu and J. R. Aranzaes, Angew. Chem., Int. Ed., 2005, 44, 7852; M. Gilhespy, M. Lok and X. Baucherel, Chem. Commun., 2005, 1085; C. M. Y. Yeung, K. M. K. Yu, Q. J. Fu, D. Thompsett, M. I. Petch and S. C. Tsang, J. Am. Chem. Soc., 2005, 127, 18010.

3 C. Moreno-Castilla and F. J. Maldonado-Hódar, Carbon, 2005, 43, 455; F. Rodríguez-Reinoso, Carbon, 1998, 36, 159.

4 G. G. Wildgoose, C. E. Banks and R. G. Compton, Small, 2006, 2, 182; A.-H. Lu and F. Schüth, Adv. Mater., 2006, 18, 1793.

5 D. Tasis, N. Tagmatarchis, A. Bianco and M. Prato, Chem. Rev., 2006, 106, 1105.

6 M. Nath, B. C. Satishkumar, A. Govindaraj, C. P. Vinod and C. N. R. Rao, Chem. Phys. Lett., 2000, 322, 333; F. L. Deepak, N. S. John, A. Govindaraj, G. U. Kulkarni and C. N. R. Rao, Chem. Phys. Lett., 2005, 411, 468.

7 Z. Yang, Y. Xia and R. Mokaya, Chem. Mater., 2005, 17, 4502; Y. Xia and R. Mokaya, Chem. Mater., 2005, 17, 1553.

8 A. Zamudio, A. L. Elías, J. A. Rodríguez-Manzo, F. López-Urías, G. Rodríguez-Gattorno, F. Lupo, M. Rühle, D. J. Smith, H. Terrones, D. Díaz and M. Terrones, Small, 2006, 2, 346; K. Jiang, A. Eitan, L. S. Schadler, P. M. Ajayan, R. W. Siegel, N. Grobert, M. Mayne, M. Reyes-Reyes, H. Terrones and M. Terrones, Nano Lett., 2003, 3, 275.

9 J. Jang and J. Bae, Angew. Chem., Int. Ed., 2004, 34, 3803; J. Jang and J. H. Oh, Adv. Mater., 2004, 16, 1650; J. Jang, B. Lim and M. Choi, Chem. Commun., 2005, 4214; J. Jang, J. H. Oh and G. D. Stucky, Angew. Chem., Int. Ed., 2002, 41, 4016.

10 J. Jang and H. Yoon, Adv. Mater., 2003, 15, 2088.

11 J. Jang and H. Yoon, Small, 2005, 1, 1195.

12 P. D. Stevens, G. Li, J. Fan, M. Yen and Y. Gao, Chem. Commun., 2005, 4435; M. Kawamura and K. Sato, Chem. Commun., 2006, 4718; S. Ko and J. Jang, Angew. Chem., Int. Ed., 2006, 45, 7564; A.-H. Lu, W. Schmidt, N. Matoussevitch, H. Bönnemann, B. Spliethoff, B. Tesche, E. Bill, W. Kiefer and F. Schüth, Angew. Chem., Int. Ed., 2004, 43, 4303; S. C. Tsang, V. Caps, I. Paraskevas, D. Chadwick and D. Thompsett, Angew. Chem., Int. Ed., 2004, 43, 5645.

13 B. Yoon and C. M. Wai, J. Am. Chem. Soc., 2005, 127, 17174.

14 Y. Li, X. M. Hong, D. M. Collard and M. A. El-Sayed, Org. Lett., 2000, 2, 2385.

15 S. U. Son, Y. Jang, J. Park, H. B. Na, H. M. Park, H. J. Yun, J. Lee and T. Hyeon, J. Am. Chem. Soc., 2004, 126, 5026. 